\title{
Spin Conductance of the Quantum Wire
}

\author{
A. DARGYS*
}

Semiconductor Physics Institute

A. Goštauto 11, 01108 Vilnius, Lithuania

\begin{abstract}
Spin transport in a semiconducting quantum wire connected to two spin-unpolarized electron reservoirs is investigated. The spin-orbit interaction is included via the Rashba Hamiltonian which together with the Zeeman Hamiltonian determines spin-filtering properties of the wire. The spin current as a function of the voltage was found to have an oscillatory or growing character.
\end{abstract}

PACS numbers: 73.21.La, 73.63.Nm, 85.35.Be, 85.75.-d

\section{Introduction}

An efficient control of electron and hole spins by electric and magnetic fields is a central theme in spintronics $[1,2]$. In nanodevices that operate in a ballistic transport regime the control can be achieved through structural asymmetry that can be manipulated by external voltage as demonstrated experimentally in Refs. $[3,4]$. In this report the spin control and filtering properties of the symmetric quantum wire (QWR) that is connected to unpolarized electron reservoirs was achieved via the spin-orbit interaction and Zeeman effect.

\section{QWR model}

The $\boldsymbol{k} \cdot \boldsymbol{p}$ Hamiltonian that describes the electron in a wire is (Fig. 1):

$$
H=(\boldsymbol{p}+e \boldsymbol{A})^{2} / 2 m^{*}+V(x)+H_{Z}+H_{R} \equiv H_{0}+H_{R},
$$

where $\boldsymbol{p}=\left(p_{x}, p_{y}\right)$ is the linear momentum and $m^{*}$ is the effective mass. The magnetic induction $\boldsymbol{B} \| z$ was included in the Zeeman Hamiltonian $H_{Z}$ as well as in the kinetic term where it appears as vector potential in the Landau gauge $\boldsymbol{A}=(0, B x, 0), B=|\boldsymbol{B}|$. The electron can freely propagate only in $y$ direction, Fig. 1. The voltage between the split gates is assumed to induce the parabolic lateral confining potential $V(x)$ in the $x$ direction, $V(x)=m^{*} \omega_{0}^{2} x^{2} / 2$, where $\omega_{0}$ is the oscillator frequency. The Zeeman and Rashba Hamiltonians are

*e-mail: dargys@pfi.lt 


$$
\begin{aligned}
& H_{\mathrm{Z}}=\frac{1}{2} g^{*} \mu_{\mathrm{B}} B \sigma_{z}, \\
& H_{\mathrm{R}}=\frac{\alpha}{\hbar}\left[\sigma_{x}\left(p_{y}+e B x\right)-\sigma_{y} p_{x}\right],
\end{aligned}
$$

where $g^{*}$ is the effective magnetic factor, $\mu_{\mathrm{B}}$ is the Bohr magneton, and $\alpha$ is the Rashba constant.

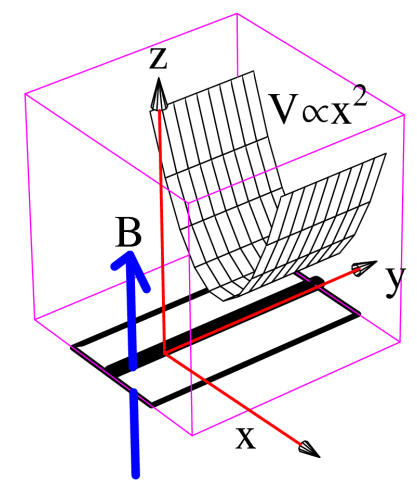

Fig. 1. The wire (thick central line) on $x-y$ plane is defined by two split gates (outer rectangles along the wire) on the $2 \mathrm{D}$ electron gas surface. The confining potential that forms the wire in $y$-direction is proportional to $x^{2}$. The magnetic field $\boldsymbol{B}$ is perpendicular to the plane.

The solution of the Schrödinger equation with the Hamiltonian (1) is separable with respect to $x$ and $y$ coordinates: $\Psi(x, y)=\psi(x) \exp \left(i k_{y} y\right)$, where $\psi(x)$ is the transverse part of the wave function and the exponent describes the running waves with the wave vectors $k_{y}$ along the wire. Substitution of $\Psi(x, y)$ into the unperturbed part $H_{0}$ of (1) yields the Hamiltonian of shifted harmonic oscillator with the effective oscillator frequency $\omega=\sqrt{\omega_{0}^{2}+\omega_{\mathrm{c}}^{2}}$ and the shift $x_{0}=\left(1+\omega_{0}^{2} / \omega_{\mathrm{c}}^{2}\right)^{-2}\left(\hbar k_{y} / e B\right)$, where $\omega_{\mathrm{c}}=e B / m^{*}$ is the cyclotron frequency. The shift is caused by magnetic field and vanishes when $B \rightarrow 0$. The eigenenergies of $H_{0}$ are

$$
E_{n( \pm)}^{(0)}=\hbar \omega\left(a_{n}^{\dagger} a_{n}+\frac{1}{2}\right)+\frac{\omega_{0}^{2}}{\omega^{2}} \frac{\hbar^{2} k_{y}^{2}}{2 m^{*}} \pm \frac{1}{2} g \mu_{\mathrm{B}} B,
$$

where $a_{n}^{\dagger}$ and $a_{n}$ are the standard raising and lowering operators, $a_{n}^{\dagger} a_{n}$ is the number operator with the eigenvalues $n=0,1,2 \ldots$, and \pm signs refer to up and down spins with respect to $\boldsymbol{B}$. The first term in (4) describes the discrete oscillator energies, the second term shows that every discrete level is associated with a parabolic band, and the last term indicates that in the magnetic field the parabolic bands are split with respect to up and down spin states. The Hamiltonian $H_{0}$ and its basis served as a starting point in finding the dispersion and spin properties of the full Hamiltonian (1) with $H_{\mathrm{R}}$ included. The calculations have 
shown that a good quantitive description of the lower energy bands of the QWR is obtained if the truncated Hilbert space characterized by a finite oscillator number $n$ is used $[5,6]$. Combining the diagonal part given by (4) and the coupling elements $\left\langle n, \nu\left|H_{\mathrm{R}}\right| m, \nu^{\prime}\right\rangle$, where $\nu$ is the spin state with respect to $z$ axis, a large Hamiltonian matrix was constructed which was then diagonalized or used to find the exact eigenfunctions numerically.

\subsection{Spin conductivity}

It is convenient to introduce three characteristic lengths: $L_{0}=\sqrt{\hbar / m^{*} \omega_{0}}$, $L_{\mathrm{Z}}=\sqrt{\hbar / m^{*} \omega_{\mathrm{c}}}$, and $L_{\mathrm{R}}=\sqrt{\hbar^{2} / 2 m^{*} \alpha}$. The oscillator length $L_{0}$ gives the spreading range of wave function in the transverse direction. The magnetic length $L_{Z}$ is equal to the radius of the skipping electron trajectory along the wire at energy $\hbar \omega_{\mathrm{c}} / 2$. For parameters of InAs $\left(m^{*} / m_{0}=0.04, \alpha=10^{-11} \mathrm{eV} \mathrm{m}, g^{*}=-8\right)$ and at $L_{0}=31 \mathrm{~nm}, B=1$ T one finds $L_{\mathrm{Z}}=25.6 \mathrm{~nm}$. The Rashba length is $L_{\mathrm{R}}=95 \mathrm{~nm}$.

The spin current is defined as a transport of electron spin in a real space. For an electron occupying a single energy band, the spin current related to spin projection $i=x$, y or $z$ can be written as

$$
I_{i}^{\mathrm{s}}=\frac{\mathrm{d}\left\langle s_{i}\right\rangle}{\mathrm{d} t}=\frac{\hbar}{2} \int_{0}^{E_{\mathrm{F}}}\left\langle\nu\left|\sigma_{i}\right| \nu\right\rangle v_{\nu}(k) f_{\nu}(k) \mathrm{d} k,
$$

where $\left\langle\nu\left|\sigma_{i}\right| \nu\right\rangle$ is the average spin in the eigenstate $|\nu\rangle$ that is to be calculated from the wire Hamiltonian, $v_{\nu}(k)$ is the electron velocity along the wire at the wave vector $k$ and $f_{\nu}(k)$ is the distribution function, which is assumed equal to one if the carrier energy is smaller than the Fermi energy $E_{\mathrm{F}}$ and zero otherwise. The interval $\left(0-E_{\mathrm{F}}\right)$ in the integral (5) can be expressed as a difference between the electro-chemical potentials $\mu_{1}$ and $\mu_{2}$ in the left and right electron reservoirs connected to the conductor: $E_{\mathrm{F}}=\mu_{1}-\mu_{2}=-e V$, where $V$ is the voltage applied between the reservoirs. In accordance with the arguments of Ref. [7], it is assumed that there is no potential drop in the channel. The potential drop that is associated with a finite resistance occurs at the connections to the reservoirs, and $V$ is supposedly divided equally between the two tapered connectors. This is essential, since the conduction can be calculated after specifying the location where the potential drop occurs. Then, the spin conductance related to a single energy subband will be

$$
G_{i}^{\mathrm{s}}=\frac{I_{i}^{\mathrm{s}}}{V}=\frac{e}{2 \pi}\left\langle\nu(\mu)\left|\sigma_{i}\right| \nu(\mu)\right\rangle .
$$

The average spin component $\left\langle\nu(\mu)\left|\sigma_{y}\right| \nu(\mu)\right\rangle$ in all cases was found to be equal to zero. This means that independent of the wave vector magnitude the average spin will be perpendicular to the wire axis. This is associated with the symmetry properties of the Hamiltonian, which in our case is real. The obtained spin conductance (6) for a single channel (subband) can be generalized to the multiband case. With the $n$-th subband having $j$ local extrema $\xi_{n}^{(j)}$, the total spin conductivity then becomes the sum over the subbands and extremal points 


$$
\mathcal{G}_{i}^{\mathrm{s}}=\mathcal{G}_{0}^{\mathrm{s}} \sum_{n, j}\left\langle\nu_{n}\left(\mu-\xi_{n}^{(j)}\right)\left|\sigma_{i}\right| \nu_{n}\left(\mu-\xi_{n}^{(j)}\right)\right\rangle \Theta\left(\mu-\xi_{n}^{(j)}\right) \operatorname{sgn}\left(m_{n}^{*(j)}\right),
$$

where $\mathcal{G}_{0}^{\mathrm{s}}=e /(2 \pi)$. The signum function accounts for a type of the extremal point. It is positive for energy minimum and negative for energy maximum. The Heaviside $\Theta$-function takes into account the open channels at a given applied voltage. Equation (7) is analogous to the quantized electrical conductance [8]. Normally, with the increase in $V$ the directions of spin in adjacent channels have opposite directions, as a result, the spin conductance, in contrast to the electrical conductance as we shall see, will have an alternating character.

\subsection{Results and discussion}

Three parts in Figs. 2-4 show the spectrum, electrical and spin conductance as a function of the voltage $V$, or equivalently of $\mu / \hbar \omega_{0}=\mathrm{eV} / \hbar \omega_{0}$, applied to reservoirs at three characteristic $\boldsymbol{B}$ magnitudes.

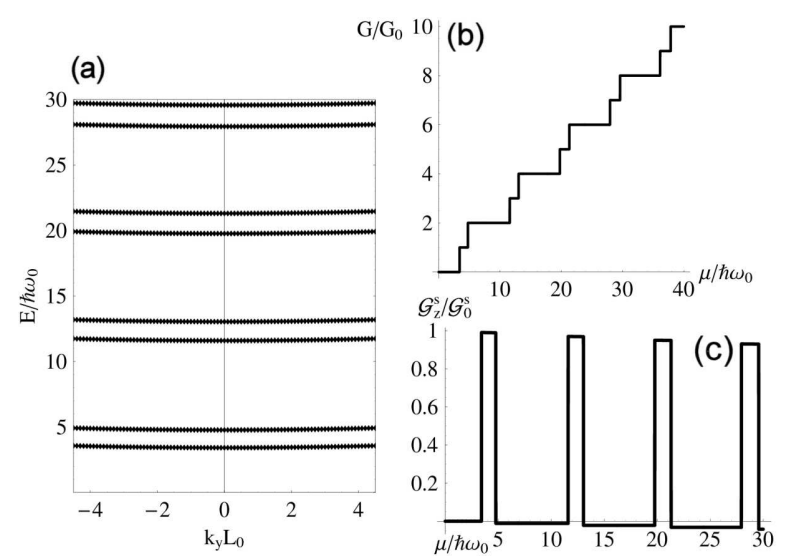

Fig. 2. (a) Spectrum and dependence of (b) electrical and (c) spin conductances on the wave vector at strong magnetic field: $L_{\mathrm{Z}} / L_{0}=0.35$ and $L_{\mathrm{R}} / L_{0}=3$. Electrical and spin normalization constants are $G_{0}=e^{2} / \hbar, \mathcal{G}_{0}^{\text {s }}=e /(2 \pi)$.

At high magnetic fields the energy bands are flat, Fig. 2a, while the adjacent subbands carry opposite spins, which are either parallel or antiparallel to $\boldsymbol{B}$. The flatness of the energy subbands can be explained by unperturbed spectrum (4), where only the second term is responsible for dispersion. In this term the effective mass $m^{*}$ has been replaced by $m^{*}\left(1+\omega_{\mathrm{c}}^{2} / \omega_{0}^{2}\right)$, which is large at high $\boldsymbol{B}$ 's. Appearance of every new step reflects the switching on of a new mode (conducting channel), where an extra electron can propagate along the wire. As a result, the electrical conductance has a stepped character. Since the total spin is equal to sum of individual spins of all open channels, while the adjacent channels carry opposite spins, the resulting spin conductance $\mathcal{G}_{i}^{\text {s }}$ consists of nearly equal amplitude pulses whose spins are parallel to $z$ axis, Fig. 2c. The pulse length is equal 


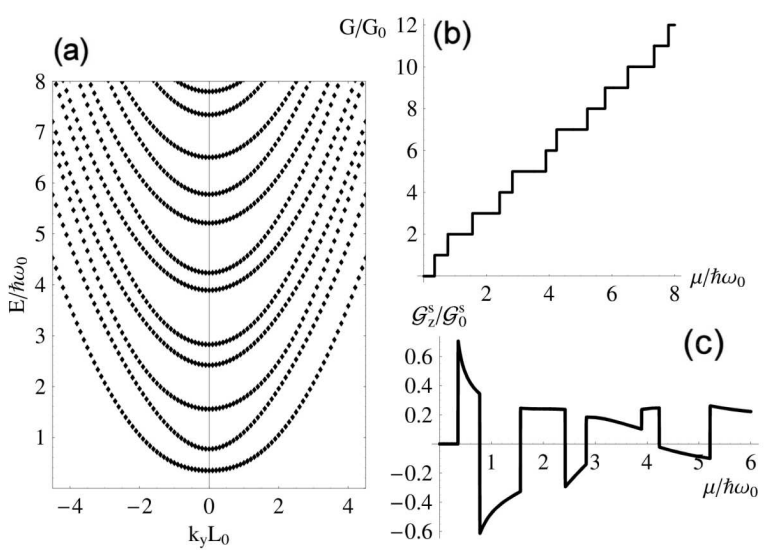

Fig. 3. The same as in Fig. 2 but at intermediate magnetic field: $L_{\mathrm{Z}} / L_{0}=1$ and $L_{\mathrm{R}} / L_{0}=1$.

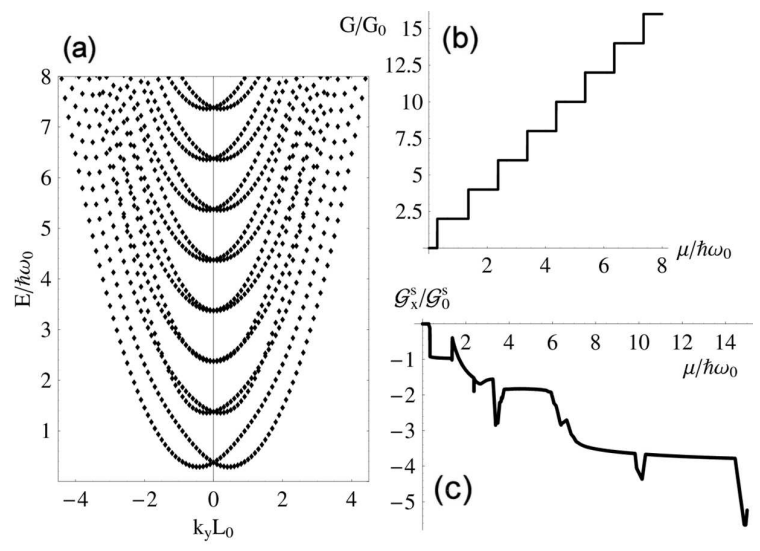

Fig. 4. The same as in Fig. 2 but at weak magnetic field: $L_{\mathrm{Z}} / L_{0}=1000$ and $L_{\mathrm{R}} / L_{0}=1$.

to energetic distance between the bands that carry opposite spins. In Fig. 2 the ratio of characteristic lengths is $L_{\mathrm{R}} / L_{0}=3, L_{\mathrm{Z}} / L_{0}=0.35$. The Rashba length in InAs is $L_{\mathrm{R}}=95 \mathrm{~nm}$. Then, at the oscillator quantum $\hbar \omega_{0}=0.02 \mathrm{meV}$ (or $L_{0}=317 \mathrm{~nm}$, the length that roughly defines the current carrying strip width in Fig. 1) the magnetic induction will be $B=0.054 \mathrm{~T}$. If the characteristic oscillator length is reduced to $L_{0}=31.7 \mathrm{~nm}\left(\hbar \omega_{0}=2 \mathrm{meV}\right)$ then one finds $B=5.4 \mathrm{~T}$.

At intermediate magnetic fields, Fig. 3, the spectrum and spin properties depend on both the Rashba and Zeeman Hamiltonians. The spin components $\left\langle\sigma_{x}\right\rangle$ and $\left\langle\sigma_{z}\right\rangle$ in this case are of a comparable magnitude and the spectrum appears to be more or less parabolic. The component $\left\langle\sigma_{z}\right\rangle$ has a pulsed character, with an abrupt changes of the spin magnitude at the crossings of the Fermi level with every higher energy subband minima. However, the compensation of the total $x$ spin 
component after switching on of a new subband in this case is not complete. In addition, due to nonvanishing contribution of the adjacent bands (channels), $\left\langle\sigma_{x}\right\rangle$ in average grows, which reflects the property that under the action of the Zeeman and Rashba Hamiltonians the spin is not in one of the pure up- or down-spin states and the average spin in different bands changes in a different manner vs. $k_{y}$.

Finally, at zero (or close to zero) magnetic field the Rashba interaction prevails, Fig. 4 . The spin splitting of the energy bands in this case is along $k_{y}$ axis rather than along energy axis as seen in Fig. 4a. In the absence of a magnetic field one has $\left\langle\sigma_{y}\right\rangle_{n}=\left\langle\sigma_{z}\right\rangle_{n}=0$ for all bands, as a result, after the switching of a new channel the $x$ component of the total spin suffers a discontinuity and the resulting spin remains nearly parallel to $x$ axis for all values of the voltage over the wire.

In conclusion, the transport of electron spin along the QWR connected to two unpolarized reservoirs were analyzed as a function of applied voltage. The total spin current along the wire (or equivalently the spin conductance) was found to be a complicated function of the voltage. Only at high magnetic fields, when the Zeeman term predominates and the spin is in a pure $\pm \hbar / 2$ state, the total spin transferred between the reservoirs has a toothed structure with teeth amplitude $\hbar / 2$. In general, due to interplay between the Rashba and Zeeman interactions, the average spin as a function of the voltage fluctuates in the plane perpendicular to the QWR.

\section{Acknowledgments}

The work was partly supported by Lithuanian State Science and Studies Foundation under contract C-07004.

\section{References}

[1] L. Worschech, D. Hartmann, S. Reitzenstein, A. Forchel, J. Phys., Condens. Matter 17, R775 (2005).

[2] W. Lu, C.M. Lieber, J. Phys. D., Appl. Phys. 39, R387 (2006).

[3] G. Engels, J. Lange, T. Schäpers, H. Lüth, Phys. Rev. B 55, R1958 (1997).

[4] D. Grundler, Phys. Rev. Lett. 84, 6074 (2000).

[5] S. Debald, B. Kramer, Phys. Rev. B 71, 115322 (2005).

[6] J. Knobbe, T. Schäpers, Phys. Rev. B 71, 035311 (2005).

[7] Y. Imry, R. Landauer, Rev. Mod. Phys. 71, S306 (1999).

[8] A.V. Moroz, C.H.W. Barnes, Phys. Rev. B 60, 14272 (1999). 